(Physiologisches Laboratorium in Bonn.)

\title{
Meine Methode der quantitativen Analyse des Glykogenes und die Arteigenthümlichkeit der Substanzen des Thierleibes.
}

\author{
Von
}

\section{Eduard Pniiger,}

Emil Abderhalden forderte mich vor einiger Zeit auf, die quantitative Analyse des Glykogenes für sein „Handbuch der "biochemischen Arbeitsmethoden" zu bearbeiten. Ich lehnte ab, weil meine Vorarbeiten zur Verbesserung der Glykogenanalyse noch nicht zum Abschluss gelangt waren. Wie ich soeben sehe, hat Dr. Karl Grube die Arbeit übernommen und in dem zweiten Bande von E. Abderhalden's Handbuch der biochemischen Arbeitsmethoden S. 159 , ohne dass ich eine Ahnung davon hatte, veröffentlicht. Da Dr. Karl Grube mehrere Jahre unter meiner Leitung in meinem Laboratorium über den Stoffwechsel der Kohlehydrate gearbeitet hat, so wird wohl vielfach vorausgesetzt werden, dass ich mit seiner Darstellung des Gegenstandes einverstanden sei. Gegen solche Voraussetzung muss ich mich verwahren! Dr. Karl Grube hat nicht einmal die wichtigen neueren Arbeiten über Glykogenanalyse, die in meinem Laboratorium ausgeführt und in diesem Archive veröffentlicht worden sind, berücksichtigt. Ich bin deshalb gezwungen, eher als es in meinem ursprünglichen Plane lag, hier eine kurze Bearbeitung meiner Methode der Glykogenanalyse zu veröffentlichen.

Die erste und wichtigste Thatsache zur Beurtheilung der in Betracht kommenden analytischen Methoden ist die Unangreifbarkeit des Glykogenes durch Kalilauge. Dieses Reagens ermöglicht deshalb die Gewinnung des Glykogenes aus den Organen des Thierleibes, weil sie diese in Lösung überführt, also das Glykogen aufschliesst, so dass es dann mit Alkohol gefällt werden kann.

Mit der Unangreifbarkeit des Glykogenes durch Kalilauge hat es aber eine besondere Bewandtniss. Denn wenn auch schon $\mathrm{Cl}$ a ude 
Bernard ${ }^{1}$ ) und August Kekule ${ }^{2}$ ) an diese Unangreifbarkeit glaubten, ergaben doch die Untersuchungen späterer Forscher, wie v. Vintschgau und Dietl ${ }^{3}$ ), R. Külz ${ }^{4}$ ) und mir selbst ${ }^{5}$ ), dass sogar verdünnte Kalilauge von 1 bis $2 \%$ das Glykogen zu zersetzen scheint. Dr. Josef Nerking ${ }^{6}$ ) gelangte sogar zu dem Ergebniss, dass die Anwendung der Kalilauge bei der Glykogenanalyse aufzugeben sei. Denn nach seiner auf zablreiche Analysen gegründeten Ansicht „wird durch den Einfluss der Kalilauge fortwährend neues "Glykogen aufgeschlossen oder abgespalten, gleichzeitig aber auch "schon gebildetes Glykogen durch die Kalilauge zerstört". Beiläufig sei hier daran erinnert, dass dureh die Untersuchungen von E. Pflüger ${ }^{7}$ ) und H. Löschke ${ }^{8}$ ) die Angaben Nerking's widerlegt sind. Denn alles Glykogen lässt sich ohne Kali und nur durch siedendes Wasser den Organen entziehen, so dass keine Berechtigung zu der Annahme vorliegt, dass das Glykogen in den Organen chemisch: gebunden sei und erst durch Kalilauge abgespalten werde.

Eine ausserordentliche Schwierigkeit für das Verständniss der Kaliwirkung entstand aber, als ich sicher nachwies, dass Glykogen durch sehr starke Kalilauge bei monatelang fortgesetztem Kochen in keiner Weise angegriffen werde. Und doch hatte ich $^{9}$ ) selbst in Uebereinstimmung mit v. Vintschgau und Dietl wie mit dem sehr exakten Arbeiter R. Külz ebenfalls Verluste beim Kochen von Glykogen mit verdünnter Kalilauge zugeben müssen.

Um eine Aufklärung der Paradoxie zu erlangen, dachte ich an die durch F. W. Pavy ${ }^{10}$ ) betonte. Thatsache, dass das nach der Methode von Brücke-Külz dargestellte Glykogen durch die Salzsüure und das Kaliumquecksilberjodid und weitere Reinigung eine Veränderung erfährt, die es für Kalilauge angreifbar macht. Die dabei entstehenden Dextrine sollen sich durch diese Eigenthümlich-

1) Claude Bernard, Leçons sur la Physiologie et la Pathologie du Système Nerveux t. 1 p. 467.

2) August Kekule, Pharmaceutisches Centralblatt 1858 S. 300.

3) v. Vintschgau und Dietl, Pflüger's Archiv Bd. 13 S. 253. 1876.

4) R. Külz, Zeitschr. f. Biol. Bd. 22 S. 161. 1886.

5) E. Pflüger, dieses Archiv Bd. 75 S. 164. 1899.

6) J. Nerking, Pflüger's Archiv Bd. 81 S. 39.

7) und 8) H. Löschke, Pflüger's Archiv Bd. 102.

9) E. PfIüger, Glykogen S. 85. Bonn 1905.

10) F. W. Pavy, The Physiology of the Carbohydrates. An Epicriticism p. 38. London 1895. 
keit auszeichnen. Ich stellte deshalb jetzt das Glykogen auf die schonendste Weise dar, indem ich es nur mit Wasser aus dem neutralisierten Organbrei oder auf andere Weise mit Ausschluss von Säuren und den Brücke'schen Reagentien auszog. Jetzt erwies sich dieses Glykogen als viel widerstandsfähiger, so dass der beim Kochen in verdünnter Kalilauge auftretende Verlust fast in die Beobachtungsfehler fiel, ja zuweilen ganz fehlte.

Ich musste also in Betracht ziehen, ob nicht das von mir auf das Sorgfältigste aus den Organen zu den Versuchen isolirte Glykogen doch eine Veränderung erfahren habe. Bereits in meinem grossen Werke über das Glykogen sagte ich: "Ich kann also mit "Sicherheit den vollkommenen Ausschluss von Fermentbildungen "nicht behaupten." Ich wollte damit sagen, dass das von mir auf das Sorgfältigste dargestellte Glykogen vielleicht doch eine Schädigung erfahren habe und nicht mehr identisch mit dem primär in den Organen enthaltenen Glykogen sei.

Da kam mir der richtige Gedanke, den frischen Organbrei in verdünnter Kalilauge beliebig lange zu kochen, aber zuletzt den Kaligehalt stark zu steigern und weiter zu kochen. Da sich nun herausstellte, dass derselbe Werth für das Glykogen erbalten wird, als wenn man gleich von Anfang an mit concentrirter Kalilauge erhitzt hätte, so ist bewiesen, dass die verdünnte Kalilauge das in den Organen befindliche Glykogen in keiner Weise versehrt. Diese wichtige Thatsache ist in einer grossen, unter meiner Leitung ausgeführten Untersuchung von Dr. Georg Francke festgestellt und in seiner Inauguraldissertation zur Erlangung der Doctorwürde der hohen veterinär-medicinischen Facultät der Universität zu Bern genauer beschrieben. Demnach gilt der Satz: Flykogen kann mit Kalilauge beliebiger Concentration beliebig lange gekocht werden, ohne dass es eine Spur von Zersetzung erfährt.

Da die wichtige Arbeit von Georg Francke ${ }^{1}$ ) sogar den Specialisten unbekannt geblieben ist, wird es zweckmässig sein, wenn ich die thatsächliche Begründung des Hauptergebnisses durch Wiederabdruck von wenigstens 2 Versuchsreihen hier mittheile.

1) Georg Francke, kgl. Kreisthierarzt in Cöln a. Rh., Ueber die Ursache, weshalb die Glykogenanalyse bei Anwendung verdünnter Kalilauge zu niedrige Werthe geliefert hat. - Bernhard Schöndorff, Peter Junkersdorf und Georg Francke, Ueber die Ursache der Fehlbeträge in der Glykogenanalyse bei Anwendung verdünnter Kalilauge. Dieses Archiv Bd. 127 S. 274. 1909. 
Meine Methode der quantitativen Analyse des Glykogenes etc.

Versuchsreihe mit Hundeleber. Doppelversuch ${ }^{1}$ ).

\begin{tabular}{|c|c|c|c|c|c|}
\hline 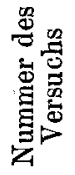 & $\begin{array}{c}\text { Concen- } \\
\text { tration } \\
\text { der } \\
\text { Kali- } \\
\text { lauge }\end{array}$ & $\begin{array}{l}\text { Koch- } \\
\text { dauer } \\
\text { in } \\
\text { Stunden }\end{array}$ & $\begin{array}{l}\text { Procent- } \\
\text { gehalt } \\
\text { an } \\
\text { Glykogen }\end{array}$ & $\begin{array}{c}\text { Mittel- } \\
\text { werthe des } \\
\text { Procent- } \\
\text { gehaltes }\end{array}$ & Bemerkungen \\
\hline $\begin{array}{l}C \\
\mathrm{D}\end{array}$ & $\begin{array}{l}30 \% \\
30 \%\end{array}$ & $\begin{array}{l}3 \\
3\end{array}$ & $\begin{array}{l}16,197 \\
16,441\end{array}$ & 16,319 & \\
\hline $\begin{array}{l}7 \\
8\end{array}$ & $\begin{array}{l}1 \% \\
1 \%\end{array}$ & $\begin{array}{l}24+1 \\
24+1\end{array}$ & $\begin{array}{l}16,686 \\
16,197\end{array}$ & 16,442 & $\begin{array}{l}\text { Nach } 24 \text { stïndigem Kochen auf } \\
30 \% \text { KOH gebracht und uoch } \\
1 \text { Stunde gekocht. }\end{array}$ \\
\hline $\begin{array}{r}9 \\
10\end{array}$ & $\begin{array}{l}1 \% \\
1 \%\end{array}$ & $\begin{array}{l}48+1 \\
48+1\end{array}$ & $\begin{array}{l}16,375 \\
16,441\end{array}$ & 16,408 & $\begin{array}{l}\text { Nach } 48 \text { stündigem Kochen anf } \\
30 \% \mathrm{KOH} \text { gebracht und noch } \\
1 \text { Stunde gekocht. }\end{array}$ \\
\hline $\begin{array}{l}11 \\
12\end{array}$ & $\begin{array}{l}1 \% \\
1 \%\end{array}$ & $\begin{array}{l}72+1 \\
72+1\end{array}$ & $\begin{array}{l}16,686 \\
16,197\end{array}$ & 16,442 & $\begin{array}{l}\text { Nach } 72 \text { stuindigem Kochen auf } \\
30 \% \text { KOH gebracht und noch } \\
1 \text { Stunde gekocht. }\end{array}$ \\
\hline
\end{tabular}

Der Doppelversuch, der aus 6 Einzelversuchen besteht, ist so angestellt, dass die $1004,6 \mathrm{~g}$ wiegende Leber eines $12,7 \mathrm{~kg}$ schweren Hundes benutzt wurde, der auf Glykogen gemästet worden war. Von derselben Leber konnten also, nachdem sie wohlzerkleinert und gemischt war, für jeden der 6 Versuche je $50 \mathrm{~g}$ Brei verwandt werden.

Versuchsreihe mit Hundemuskel. Doppelversuche ${ }^{2}$ ).

\begin{tabular}{|c|c|c|c|c|c|}
\hline 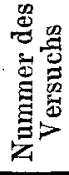 & $\begin{array}{l}\text { Concen- } \\
\text { tration } \\
\text { der } \\
\text { Kali- } \\
\text { lange }\end{array}$ & $\begin{array}{l}\text { Koch- } \\
\text { dauer } \\
\text { in } \\
\text { Stunden }\end{array}$ & $\begin{array}{l}\text { Procent- } \\
\text { gehalt } \\
\text { an } \\
\text { Glkyogen }\end{array}$ & $\begin{array}{c}\text { Mittel- } \\
\text { werthe des } \\
\text { Procent- } \\
\text { gehaltes }\end{array}$ & Bemerkungen \\
\hline $\begin{array}{l}\mathrm{G} \\
\mathrm{H}\end{array}$ & $\begin{array}{l}30 \% \\
30 \%\end{array}$ & $\begin{array}{l}3 \\
3\end{array}$ & $\begin{array}{l}2,034 \\
2,048\end{array}$ & 2,041 & \\
\hline $\begin{array}{l}23 \\
24\end{array}$ & $\begin{array}{l}1 \% \\
1 \%\end{array}$ & $\begin{array}{l}24+1 \\
24+1\end{array}$ & $\begin{array}{l}2,034 \\
2,106\end{array}$ & 2,0699 & $\begin{array}{l}\text { Nach } 24 \text { stündigem Kochen auf } \\
30 \% \text { KOH gebracht und noch } \\
1 \text { Stunde gekocht. }\end{array}$ \\
\hline 25 & $1 \%$ & $48+1$ & 2,106 & 2,106 & $\begin{array}{l}\text { Nach } 48 \text { stündigem Kochen wie } \\
\text { Nr. } 23 \text { und } 24 \text { behandelt. }\end{array}$ \\
\hline $\begin{array}{l}26 \\
27\end{array}$ & $\begin{array}{l}1 \% \\
1 \%\end{array}$ & $\begin{array}{l}72+1 \\
72+1\end{array}$ & $\begin{array}{l}2,1105 \\
1,9499\end{array}$ & 2,030 & $\begin{array}{l}\text { Nach } 72 \text { stündigem Kochen auf } \\
30 \% \mathrm{KOH} \text { gebracht und noch } \\
1 \text { Stunde gekocht. }\end{array}$ \\
\hline
\end{tabular}

1) Dieses Archiv Bd. 127 S. 276.

2) Dieses Archiv Bd. 127 S. 277. 
Es kann also kein Zweifel sein, dass diejenige Art des Glykogenes, welches primär in den Organen enthalten ist, durch verdünıte Kalilauge nicht angegriffen wird.

Dr. Karl Grube sagt aber in seiner soeben erst erschienenen AbhandJung vom Glykogen:

„Mit starker Kalilauge kann es gekocht werden, ohne dass es "sich zersetzt, durch schwache wird es angegriffen."

Will man sich Rechenschaft ablegen über die Ursache, weshalb bei Anwendung verdünnter Kalilauge auf Glykogen grössere Verluste bisher beobachtet worden sind als beim Erhitzen mit concentrirter Kalilauge, so kommen wesentlich zwei Umstände in Betracht.

1. Wenn das Organ durch Kochen mit verdünnter Lauge in Lösung gebracht wurde, ist die Denaturirung des Eiweisses nicht weit genug vorgeschritten. Es sind also noch viele Eiweissflocken da, welche Glykogen einschliessen, so dass es nicht ausgewaschen werden kann. - Bei Kochen mit concentrirter Lauge ist das Eiweiss denaturirt, so dass eine viel kleinere Menge von kleineren Flocken erhaiten worden ist, die kein Glykogen mehr einschliessen. - Ieh habe ja quantitativ nachgewiesen, dass bei der Methode von $\mathrm{K} \ddot{u} \mathrm{lz}$ nach Anwendung der verdünnten Kalilauge oft ungeheuer grosse Glykogenmengen ${ }^{1}$ ) so in den Eiweissniederschlag eingebacken sind, dass sie nicht ausgewaschen werden können.

2. Die zweite Ursache zu Glykogenverlust bat ihren Grund darin, dass das Glykogen, wenn es aus dem Organe isolirt worden ist, durch die zur Isolation verwandte chemische Behandlung oder auch durch einwirkende Fermente eine Veränderung erfahren hat.

3. Nicht ausreichend aufgeklärt ist meine sonderbare Beobachtung, dass das angreifbar gewordene Glykogen bei Behandlung mit concentrirter Kalilauge sich als resistent erweist. Ich beabsichtige diesen paradoxen Punkt nochmals eingehend zu prüfen. -

Nachdem festgesteilt war, dass thatsächlich die Ausbeute an Glykogen immer zu klein ausfällt, wenn verdünnte Kalilauge zur Aufschliessung der Organe aufgewandt wird, war es notwendig festzustellen, wie gross die Concentration der Kalilauge sein muss, um richtige Werthe $\mathrm{zu}$ erhalten. Mit dieser Untersuchung habe ich Herrn Paul Heyden beauftragt, welcher dieselbe unter meiner

1) E. Pflüger, Glykogen S. 39. Bonn 1905. 
Leitung in meinem Laboratorium bearbeitet und in seiner Inauguraldissertation ${ }^{\mathbf{l}}$ ) veröffentlicht hat.

Bei der Vergleichung der Wirkung der $30 \%$ igen und $2 \%$ igen Lauge ergab sich ein sofort in die Augen springender grosser Fehlbetrag bei Anwendung der verdünnten Lauge.

Auffallend erscheint es aber, dass bei Vergleichung der $30 \%$ Kalilauge mit der von $\mathrm{J} 5 \%$ oder $7,5 \%$, zwar auch noch die reichere Ausbeute bei $30 \%$ sicher heraustritt, aber doch ist der Unterschied meist kleiner, ja fehlt ganz.

Da ein Unterschied aber auch bei Vergleichung von $30 \%$ iger mit 15\% iger Kalilauge in gedachter Richtung noch deutlich bemerkbar bleibt, folgt, dass die richtige Stärke der Kalilauge $30 \%$ beträgt.

Es blieb nun noch die Frage zu erörtern, wie lange die Organe mit $30 \%$ iger Kalilauge gekocht werden müssen, um die vollständige Zerstöruug der Eiweissstoffe zu erzielen. Diese Aufgabe zu behandeln habe ich Herrn Victor Hessen ${ }^{2}$ ) übergeben, der seine Ergebnisse, die er unter meiner Leitung in meinem Laboratorium erhielt, in seiner Inauguraldissertation veröffentlicht hat.

Es geht aus diesen Versuchen hervor, dass man dieselben Glykogenwerthe erbält, wenn man die Organe $1 / 2,1,2$ oder 3 Stunden mit $30 \%$ Kalilauge kocht; eine halbstündige Kochdauer genügt also, wenn man nur dafür Sorge trägt, dass der Kolben alle 5 bis 10 Minuten aus dem Wasserbade herausgenommen und geschüttelt wird.

Die Begründung folgt aus diesen Tabellen:

1) Paul Heyden, prakt. Thierarzt in Hermülheim bei Cöln a. Rh., Ueber den Einfluss, den die Concentration der Kalilauge auf die quantitative Analyse des Glykogenes ausübt. Der veterinär-medicinischen Facultät in Giessen vorgelegt. Dasselbe kürzer abgefasst von Bernhard Schöndorff, Peter Junkersdorf, Paul Heyden. Dieses Archiv Bd. 126 S. 582.1909.

2) Victor Hessen, städtischer Thierarzt in Barmen, Ueber den Einfluss, den die Zeit der Erhitzung mit starker Kalilauge auf die quantitative Analyse des Glykogenes ausübt. Der hohen veterinär-medicinischen Facultät der Universität Bern vorgelegt. 1909. - Dasselbe in abgekürzter Form von Bernhard Schöndorff, Peter Junkersdorf, Victor Hessen. Dieses Archiv Bd. 126 S. 578.1909. 
Tabelle.

\begin{tabular}{|c|c|c|c|c|c|}
\hline \multirow{2}{*}{$\begin{array}{l}\text { Nummer } \\
\text { der Ver- } \\
\text { suchsreihe }\end{array}$} & \multirow{2}{*}{$\begin{array}{c}\text { Untersuchtes } \\
\text { Organ }\end{array}$} & \multicolumn{4}{|c|}{$\begin{array}{c}\text { Mittelwerthe des Glykogengehaltes in Procenten } \\
\text { nach Kochdauer in Stunden: }\end{array}$} \\
\hline & & 3 & 2 & 1 & $1 / 2$ \\
\hline I & Muskel & 0,3169 & - & 0,3223 & 一 \\
\hline II & $n$ & 0,885 & - & 0,8775 & - \\
\hline III & $"$ & 0,934 & 0,923 & 0,92 & - \\
\hline IV & 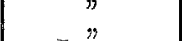 & 1,185 & 1,212 & 1,292 & 1,185 \\
\hline V & Leber & 13,568 & 13,282 & 13,428 & 13,401 \\
\hline VI & Muskel & 2,4075 & 2,458 & 2,4198 & 2,4708 \\
\hline VII & Leber & 17,7628 & - & 17,8339 & 17,6914 \\
\hline VIII & Muskel & 3,897 & - & 3,85 & 3,897 \\
\hline
\end{tabular}

Tabelle.

\begin{tabular}{|c|c|c|}
\hline & $\begin{array}{l}\text { Kochdauer } \\
3 \text { Stunden }\end{array}$ & $\begin{array}{l}\text { Kochdaner } \\
1 \text { Stunde }\end{array}$ \\
\hline \multirow{3}{*}{$\begin{array}{l}\text { Mittel aus den Muskelwerthen von Versuchs- } \\
\text { reihen I, II, III, IV, VI und VIII ... } \\
\text { Mittel aus den Leberwerthen von Versuchsreihe } \\
\text { V und VII. . . . . . . . . . . }\end{array}$} & $1,6209 \%$ & $1,6036 \%$ \\
\hline & $15,6654 \%$ & $15,6808 \%$ \\
\hline & $\begin{array}{l}\text { Kochdauer } \\
3 \text { Stunden }\end{array}$ & $\begin{array}{l}\text { Kochdauer } \\
2 \text { Stunden }\end{array}$ \\
\hline \multirow[t]{2}{*}{$\begin{array}{l}\text { Mittel aus den Muskelwerthen von Versuchs- } \\
\quad \text { reihe III, IV, VI . . } \cdots \cdots\end{array}$} & $1,5088 \%$ & $1,564 \%$ \\
\hline & $\begin{array}{l}\text { Kochdaner } \\
3 \text { Stunden }\end{array}$ & $\begin{array}{l}\text { Kochdauer } \\
1 / 2 \text { Stunde }\end{array}$ \\
\hline $\begin{array}{l}\text { Mittel aus den Muskelwerthen von Versuchs- } \\
\text { reihe IV, VI, VIII . . . . . . . . }\end{array}$ & $2,4965 \%$ & $2,5176 \%$ \\
\hline $\begin{array}{l}\text { Mittel aus den Leberwerthen von Versuchsreihe } \\
\text { V und VII. . . . . . . . }\end{array}$ & $15,6636 \%$ & $15,546 \%$ \\
\hline
\end{tabular}

Weil aber das häufige Herausnehmen des Kolbens aus dem siedenden Wasserbad äusserst lästig ist, verfahren wir gewöhnlich so, dass wir dies nur im Anfange ein- oder zweimal vollziehen, jedesmal gut schütteln und dann 2 Stunden erhitzen. Das hat auch den grossen Vortheil, dass die ausreichende Zerstörung der Gewebe selbst dann sicher erzielt wird, wenn es sich um die Aufschliessung der Organe sehr alter Thiere handelt. Es ist z. B. sehr auffallend 
und von mir oft beobachtet, dass besonders bei Anwendung verdünnter Kalilauge die Lösung der Organe sich in ausserordentlicher Weise verzögert, wenn es sich um sehr alte Thiere handelt.

Demgemäss verfahre ich nunmehr so: $100 \mathrm{~g}$ mit der Fleischhackmaschine zerkleinertes Organ wird eingetragen in einen Kolben, welcher bereits $100 \mathrm{ccm}$ Kalilauge von $60 \%$ enthält und schon längere Zeit im siedenden Wasserbad auf $100^{\circ} \mathrm{C}$. erhitzt worden ist. Hierdurch wird die im Organbrei sich vollziehende Fermentation sofort unterbrochen. Nachdem der Kolben 10 Minuten im siedenden Bad war, wird er herausgehoben, geschüttelt und dann wieder in das siedende Bad zurückgebracht. Das wird dann 20 Minuten nach Beginn der Erhitzung wiederholt. - Sobald die Erhitzung 2 Stunden gedauert hat, wird der Kolben aus dem Wasserbad gehoben, unter der Pumpe durch einen continuirlichen Wasserstrahl abgekühlt und in ein Becherglas entleert. Mit $200 \mathrm{ccm}$ Wasser wird der Kolben rein gespült, so dass die Lösung auf ungefähr 100 cem gebracht wird, die man dann mit $800 \mathrm{ccm}$ Alkohol von $96 \%$ Tr. versetzt, gut umrührt und über Nacht mit einem Uhrglas verschlossen hinstellt, damit sich das Glykogen gut absetze.

Von dem grossen Becherglas wird nun die über dem Niederschlag stehende Flüssigkeit in ein zweites geräumiges Becherglas abgegossen und sofort mit Hilfe eines sehr grossen mit Hahn versehenen Trichters durch ein gutes Faltenfilter abfiltrirt. -

Der Glykogenniedersehlag wird auf ein zweites schwedisches Filter gebracht, welcher einen Durchmesser von $15 \mathrm{~cm}$ besitzt. Dieser Niederschlag wird mit $66 \%$ igem Alkohol so lange gewaschen, bis das Filtrat nahezu farblos abläuft. Dann folgt Waschung mit Alkohol absolutuş, dann mit Aether und zuletzt nochmals mit absolutem Alkohol.

Das Faltenfilter, welches die decantirte Flüssigkeit aufgenommen hatte, wird nur mit $66 \%$ igem Alkohol gewaschen.

Es handelt sich jetzt darum, das Glykogen zu trennen von den Flocken und Farbstoffen, die es noch verunreinigen. Ich nehme das feuchte schwedische Filter vom Trichter, entfalte dasselbe über einem geräumigen Becherglas von ca. 300 bis $500 \mathrm{ccm}$ Gehalt und 
streiche mit einem Platinspatel die ganze Schmiere vom Papiere ab, so dass sie in das Becherglas fällt. Dann bringe ich das Filter wieder auf den Trichter und befestige das Faltenfilter über das Filter, von dem das Glykogen abgewischt wurde. Es stehen also zwei Filter übereinander. Nunmehr giesse ich siedendes Wasser auf das Faltenfilter, so dass das Filtrat das untere Filter füllt und die noch anhaftenden Glykogenspuren löst, was man durch ein Pinselchen befördert. So erhält man das ganze Glykogen mit seinen Verunreinigungen in das Bechergläschen. Durch längeres Umrühren bringt man das Glykogen in Lösung und filtriert dann durch Glaswolle in ein geaichtes Kölbchen von 100 bis 200 bis 500 bis $1000 \mathrm{cem}$ Gehalt. Meist genügt ein Kölbchen von $200 \mathrm{ccm}$. Sind grössere Glykogenmengen vorbanden, muss man geräumigere Kolben verwenden. Die trübe schmutzige Lösung wird nun in dem noch nicht ganz aufgefüllten Kölbchen abgeküh]t und mit ein wenig Salzsäure von 1,19 spec. Gew. versetzt. Man tröpfelt vorsichtig $z u$, bis sich deutliche Flöckchen ausscheiden, welche durch klare Flüssigkeit geschieden sind. Gewöhnlich gebraucht man 0,5 bis 1 bis $2 \mathrm{ccm}$ bei Untersuchung von $100 \mathrm{~g}$ Organ. Giesst man zu viel oder zu wenig Salzsäure zu, so erhält man kein klares Filtrat. Man muss hier langsan mit grosser Vorsicht die kleinen Zusätze machen. Glaubt man die richtige Fällung erzielt zu haben, so füllt man das Kölbchen auf bis zur Marke, giesst in ein Becherglas aus und wartet einige Zeit, bis die meist stark gefärbten Flocken sich gesenkt haben. Dann filtrirt man entweder durch ein schwedisches Filter oder durch "Blauband" Nr. 589 aus der Fabrik von Schleicher \& Schüll in Düren, wenn das schwedische Filter kein ganz klares Filtrat liefert.

Dieses Verfahren hat nun den grossen Vorzug, dass das Filtrat sofort mit dem Polarisationsapparat auf seinen Gehalt an Glykogen untersucht werden kann. Ich werde dem Leser den Beweis liefern, dass diese Methode von unvergleichlicher Genanigkeit ist und ausserordeutlich schnell zum Ziele führt, obwohl es sich um eine quantitative Methode handelt.

Das Merkwürdige ist, dass bei richtiger Ansäuerung der unreinen Glykogenlösung ein vollkommen farbloses Filtrat erhalten wird, das sich zur Polarisation ausgezeichnet eignet. Es ist wahr, dass zuweilen eine Färbung vorhanden ist, die mich aber fast niemals verhinderte, die Ablesung machen zu können, nachdem ich entsprechend verdünnt hatte. Wenn ich das Glykogen aus besonderen Gründen 
mit $10^{\circ}$ Vol. Alkohol gefällt hatte, machte sich der Farbstoff mit grösserer Entschiedenheit wie sonst störend geltend.

Die durch die schwache Ansäuerung der rohen Glykogenlösung erzeugten flockigen Niellerschläge sind von sehr verschiedener Beschaffenheit, aber immer nur von geringem Gewicht, wenn sie auch nach der Fällung durch ihr aufgequollenes Volum als grosse Masse imponịren. Beim Abfiltriren dieser Fällungen sieht man, dass es sich nur um dünne Ueberzüge auf dem Filtrierpapier handelt. Die Fällungen aus der Leberglykogenlösung sind immer stárk gefärbt, und zwar sehr versechieden, so dass orangerothe, gelbe und schwarze vorkommen, die sich auch durch ihre Löslichkeit in Alkohol von $66 \%$ und von Alcohol absolutus unterscheiden. Meist sind diese Farbstoffe in Aether unlöslich; doch ist dies nicht immer der Fall.

Stets ist ein grosser Theil der Farbstoffe in vèrdünutem Alkohol von $66 \%$ und in absolutem Alkohol wie in Aether ganz unlöslich. -

Die bei der Glykogenanalyse der Muskeln anftretenden. Farbstoffe sind immer mit der Leber verglichen in sehr geringer Menge vorhanden.

Bei grossem Reichthum an Glykogen ist die Menge der Verunreinigungen absolut und relativ viel geringer, und die Filtration vollzieht sich beim Auswaschen mit Alkohol sehr schnell und leicht.

Dass die Farbstoffflocken bei der Filtration keinen Fehler durch Zurückhaltung von Glykogen bedingen, habe ich schon früher streng bewiesen ${ }^{1}$ ). -

Ich gebe nunmehr zuerst eine Tabelle einer grossen Zahl yon Glykogenanalysen des Froschkörpers. Stets wurden neun bis zehn Frösche gleichzeitig in Arbeit genommen, so dass also das Gesammtglykogen des ganzen Körpers zur Untersuchung kam. "Dadurch werden "die individuellen Schwankungen" des Glykogengehaltes möglichst eliminirt. Das Genauere über die Einzelheiten der Tabelle findet sich in einer von mir bereits veröffentlichten Abhandlung, die ich hier aus bald ersichtlichen Gründen nochmals vorlegé.

1) Eduard Pflüger, Unter gewissen Lebensbedingungen nimmt die 'in dem lebendigen Thierkörper enthaltene Menge des Glykogenes trotz vollkomméner über Monate sich ansdehnender Entziehung der Nahrung fơrtwährend sehr erheblich zu. Dieses Archiv Bd. 120 S. 285. 1907. 


\begin{tabular}{|c|c|c|c|c|}
\hline \multirow{2}{*}{$\begin{array}{c}\text { Zabl } \\
\text { der } \\
\text { Frösche }\end{array}$} & \multirow{2}{*}{$\begin{array}{l}\text { Gewicht } \\
\text { der } \\
\text { Frösche }\end{array}$} & \multicolumn{2}{|c|}{$\begin{array}{l}100 \mathrm{~g} \text { Frösche enthalten } \\
\text { Glylogen in Gramm gemäss }\end{array}$} & \multirow{2}{*}{$\begin{array}{l}\text { Absoluter } \\
\text { Unterschied }\end{array}$} \\
\hline & & Polarisation & Titration & \\
\hline
\end{tabular}

Weibliche Frösche.

\begin{tabular}{r|c|c|c|c}
10 & 513,0 & 0,423 & 0,417 & 0,06 \\
10 & 477,5 & 0,458 & 0,465 & 0,07 \\
10 & 566,0 & 0,486 & 0,486 & 0,00 \\
10 & 535,0 & 0,550 & 0,549 & 0,01 \\
9 & 479,0 & 0,557 & 0,561 & 0,04 \\
10 & 608,5 & 0,620 & 0,643 & 0,23 \\
10 & 519,5 & 0,632 & 0,625 & 0,07 \\
10 & 510,5 & 0,659 & 0,653 & 0,06 \\
10 & 451,0 & 0,600 & 0,609 & 0,09 \\
\hline Summa & - & 4,985 & 5,008 & -
\end{tabular}

Männliche Frösche.

\begin{tabular}{r|c|c|c|c}
10 & 492,5 & 0,632 & 0,639 & 0,07 \\
10 & 410,0 & 0,626 & 0,589 & 0,37 \\
5 & 196,5 & 0,733 & 0,749 & 0,16 \\
10 & 441,0 & 0,719 & 0,709 & 0,10 \\
9 & 416,0 & 0,719 & 0,689 & 0,30 \\
9 & 345,0 & 0,872 & 0,867 & 0,05 \\
\hline Summa & - & 4,301 & 4,242 & -
\end{tabular}

3 männliche +3 weibliche Frösche (Esculentae).

\begin{tabular}{c|c|c|l|c}
10 & 492,5 & 0,632 & 0,639 & 0,07 \\
10 & 510,0 & 0,755 & 0,7897 & 0,34 \\
10 & 437,5 & 0,838 & 0,820 & 0,18 \\
10 & 513,0 & 0,4235 & 0,417 & 0,06 \\
10 & 600,0 & 0,607 & 0,609 & 0,02 \\
10 & 483,0 & 0,828 & 0,812 & 0,16 \\
\hline Summa & - & 4,086 & 4,087 & -
\end{tabular}

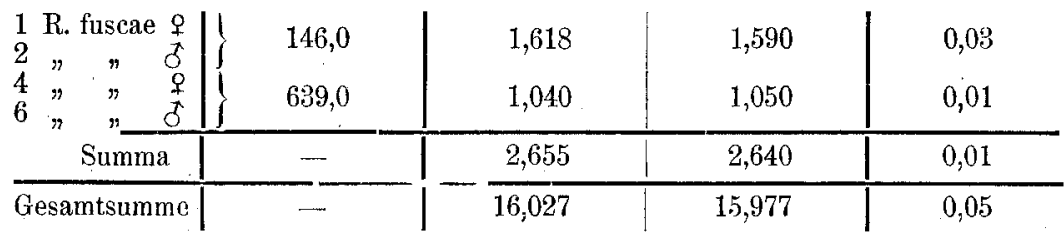

Da die Zahl der Fälle $=23$, so ist das allgemeine Mittel:

\section{$0,6965 \quad 0,6947$}

Die Tabelle beweist für das Froschglykogen, dass ausser dem Glykogen kein drehender Körper vorhanden war und nach Invertirung des ülykogenes neben dem Zucker keine Substanz, welche reducirend wirkte.

Gleichwobl ist es vorgekommen, dass in einem Ausnahmefall die Polarisation einen um $14 \%$ höheren Werth als die Titration 
ergab. Eingehende Wiederholung des Versuches bestätigte die Thatsache, so dass die Annahme einer abnormen, das Glykogen begleitenden Substanz kaum bezweifelt werden konnte.

Bei diesen polarimetrischen Bestimmungen des Glykogenes hat man es im Allgemeinen mit Ablesungen von $2^{0}$ zu thun; meistens handelt es sich um geringere Werthe. Soviel ich gesehen habe, unterseheiden sich die Ablesungen der besten Beobachter un $0,01^{\circ}$ bis $0,03^{\circ}$. Ist also die correcte Ablesung $1,000^{\circ}$, so wird ein Fehler bis $3 \%$ begangen. Bei manchen Beobachtern ist aber der Fehler noch viel grösser. Es lässt sich ja natürlich durch eine sehr grosse Zahl von Ablesungen ein der Wahrheit entsprechendes Mittel erzielen, wie es bei Bestimmung der specifischen Drehung durchgeführt werden muss. Bei den hier mitgetheilten Analysen war ein so hoher Grad von Genauigkeit nicht geboten. Ich habe mich mit drei bis sechs Ablesungen begnügt, jedesmal aber die Ablesungen mit der Normalquarzplatte controllirt.

Hervorheben muss ich, dass alle Analysen von mir selbst ausgeführt worden sind, ganz ohne jede. Beihülfe eines Assistenten, was der Gleichmässigkeit der Beobachtung halber nöthig war.

Ich wende mich jetzt zu Glykogenanalysen, die nur an Hunden ausgeführt worden sind, bei denen nur die Leber oder die Muskeln untersucht wurden. Bei dieser sehr ausgedehnten Untersuchung habe ich selbst alle Glykogenuntersuchungen mit dem Polarisationsapparat ausgeführt, während mein chemischer Assistent Herr Dr. Peter Junkersdorf nach Invertirung des Glykogenes den Zucker nach Fehling-Soxhlet titrirte.

Es handelt sich in der That um eine Auslese aus sehr vielen verschiedenartigen Untersuchungen, so dass die Ernährung der Hunde sehr verschieden sich gestaltete. Viele von den Hunden waren vorher mit Phloridzindosen diabetisch gemacht worden. Das Alles soll hier nicht in Betracht gezogen werden, weil es sich nur darum handelt, zu beweisen, dass unter den verschiedensten Ernährungsbedingungen meine Methode der quantitativen Analyse in äusserst kurzer Zeit obsolut sichere Werthe liefert. Die Auslese der Analysen unterlag natürlich der strengen Bedingung, dass alle Analysen mitgetheilt sind, bei denen sowohl die Polarisation wie die Titration angewandt worden ist. 
Tabelle.

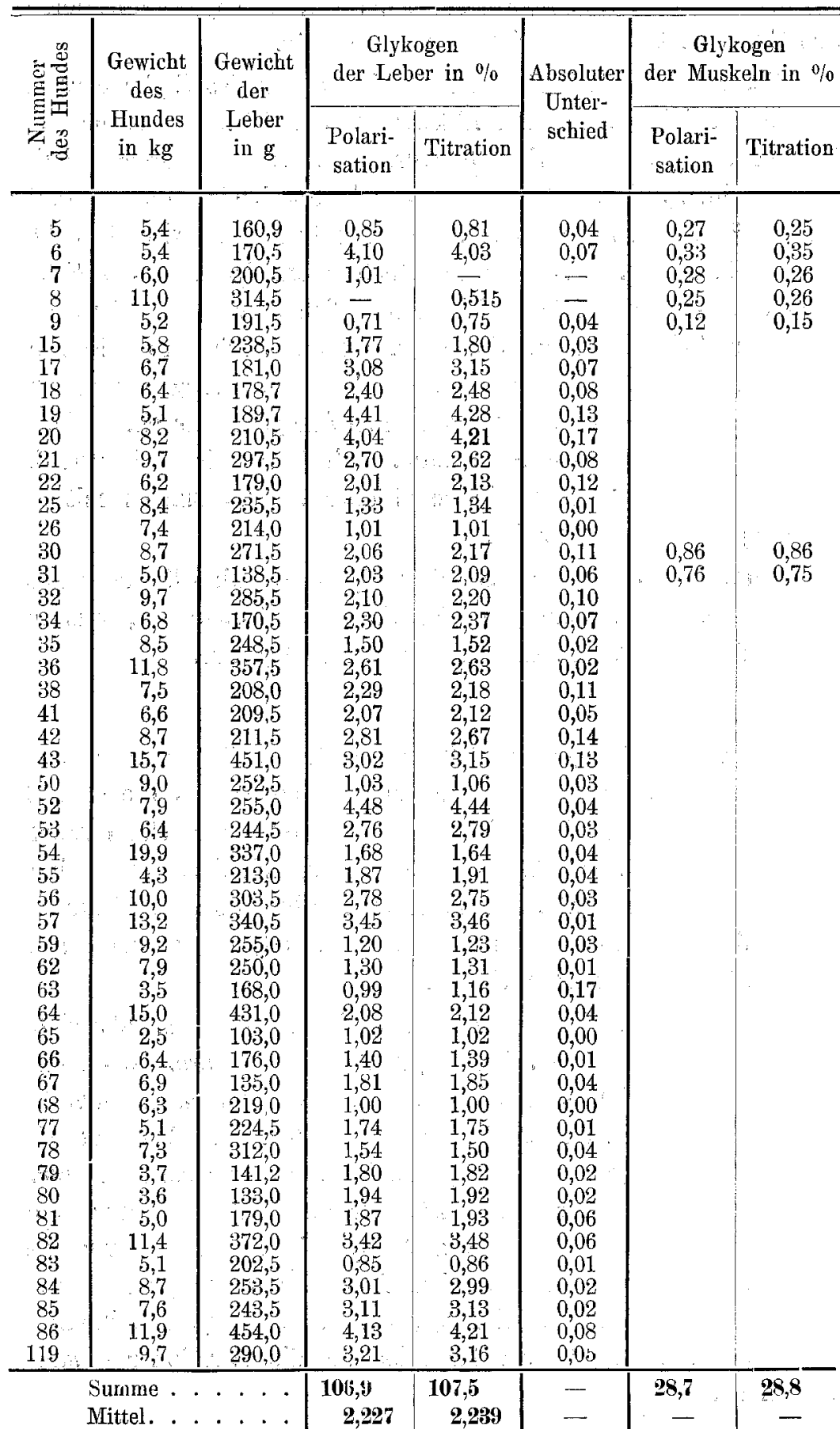


Diese Untersuchungen beweisen also, dass durch die Polarisation der Glykogengehalt schnell und richtig gefunden wird. Nur in sehr seltenen Fällen stimmt die Bestimmung durch Polarisation nicht ganz mit der Titration nach Fehling-Soxhlet überein. Sehr gross sind die Unterschiede aber auch nicht. - Wünschenswerth bleibt deshalb die Controlle der Polarisation durch die Titration.

Weil man dureh die Polarisation sehr annähernd den Gehalt an Zucker kennt, der durch Invertirung der Glykogenlösung entstehen muss, führt die Titration meist in wenigen Minuten $\mathrm{zu}$ einem sicheren Ergebniss, während die gravimetrische Methode immer einige Stunden erfordert. Ich habe deshalb die gravimetrische Methode mit Volhard's Berichtigung nur angewandt, wo der Farbstoffgehalt der Glykogen- und Zuckerlösung die polarimetrische und Fehling sehe Bestimmung unsicher oder unmöglich machte, oder wo die zu kleine zu bestimmende Zuckermenge die Titration nicht mehr ausführbar erseheinen liess. -

Demnach gestaltet sich die Analyse folgendermaassen: Ich fülle ein Rohr von 189,4 mm Länge mit der zu untersuchenden Glykogenlösung und mache am Halbschattenapparat drei Ablesungen

$$
\begin{array}{rr} 
& +2,10^{\circ} \\
& +2,08^{\circ} \\
& +2,08^{\circ}
\end{array}
$$

Corrigirt: $+2,066^{\circ}$

Weil nun die specifische Drehung der Dextrose $=+52,60^{\circ 1}$ ) und die des Glykogenes $=+196,57^{\circ 2}$ ), so hat man

$$
\frac{196,57^{\circ}}{52,60^{\circ}}=3,74 \text { und } \frac{2,066^{\circ}}{3,74}=0,552 \text { Glykogen. }
$$

Von dieser Glykogenlösung. werden nun $90 \mathrm{ccm}$ in einem 150 ccm-Kölbchen mit 5 ccm Salzsäure von 1,19 spec. Gew. 3 Stunden erhitzt, abgekühlt mit $60 \%$ iger Kalilauge neutralisirt, bis zur Marke aufgefüllt und durch schwedisches Filter filtrirt, weil sich noch einige Farbstofflöekchen ausgeschieden haben.

1) H. Landolt, Das optische Drehungsvermögen organischer Substanzen, II. Aufl., S. 446. 1898.

2) Mme Gatin-Gružewska, Das reine Glykogen. Dieses Archiv Rd. 102 S. 577. 1904 . 
Invertirt wurden $9 / 10$ des in $100 \mathrm{ccm}$ gelösten Glykogenes, also $0,552-0,0552=0,4968 \mathrm{~g}$ Glykogen.

Nun hat Nerking durch eine genaue Untersuchung: folgende Gleichung festgestellt:

$$
\text { Glykogen }=\text { Zucker } \times 0,927 .
$$

Daraus folgt, dass 0,4968 Glykogen liefern müssen 0,5359 Dextrose, welche wir in $150 \mathrm{ccm}$ gelöst hatten, so dass die Lösung $0,3573 \%$ Dextrose enthält.

Zur Analyse nach Fehling-Soxhlet wurden 10 cem Kupferseignettesalzlösung $+40 \mathrm{ccm}$ Wasser gebraucht, für deren vollkommene Reduction $0,050 \mathrm{~g}$ Dextrose in runder Zahl nöthig sind. Weil unsere Zuckerlösung gemäss des durch Polarisation bestimmten Werthes $0,3573 \mathrm{~g}$. Dextrose in $100 \mathrm{ccm}$ enthält, würden rund $14 \mathrm{ccm}$ dieser Zuckerlösung nöthig sein, um die $10 \mathrm{ccm}$ Fehling vollkommen zu reduciren. Bei der ersten Analyse und Zusatz von $14 \mathrm{ccm}$ Zuckerlösung gab das Filtrat der 2 Minuten gekochten Mischung noch eine Spur Reaction mit Ferrocyankalium; bei Anwendung von $14,2 \mathrm{ccm}$ reagirte das Filtrat nicht mehr auf dies Reagens. Also 14,1 ccm $=0,05 \mathrm{~g}$ Dextrose, also $100 \mathrm{ccm}=0,355$ und $150 \mathrm{~cm}=0,533$. Da dies nur $9 / 10$ der Gesammtmenge, so beträgt diese $0,592 \mathrm{~g}$ Dextrose, entsprechend 0,549 Glykogen.

Also :

Gemäss Polarisation: $0,552 \mathrm{~g}$ Glykogen, \#Titration: 0,549 , ”

Der Unterschied beträgt absolut:

$$
0,003 \text { g Glykogen. }
$$

Bei dem vorliegenden Versuch entsprachen:

$113 \mathrm{~g}$ Frosch $=0,552 \mathrm{~g}$ Glykogen oder

$100 " n=0,488$ " ,

Betreffend die gravimetrische Methode verweise ich auf mein Werk: Glykogen (S. 53 und 111). -

Ich möchte endlich noch auf den Vorzug meiner Methode aufmerksam machen, welcher es ermöglicht, die kleinsten Mengen von Glykogen quantitativ genau zu bestimmen, selbst da noch, wo die Titration, ja die gravimetrische Analyse versagen. Ich bestimme mit meinem. Polarimeter noch Werthe von 0,05 bis 0,01 g Glykogen auf $100 \mathrm{~g}$ Leber oder Muskel, und ich habe von diesem Vortheil 
vielfachen Gebrauch gemacht. - Sehr of t wird man bei der Seltenheit der Incongrurenz $z$ wischen Polarisation und Titrationaf Inversion und Titrationzurgrossen Abkürzung verzichten dürfen!

Schliesslich möchte ich noch eiumal auf ein Ergebniss dieser Untersuchung zurückkommen, das ich für äusserst wichtig ansehe. Es hat sich als sicher herausgestellt, dass das aus dem Körper der Frösche isolirte Glykogen derselbe Stoff ist, wie das aus der Leber oder den Mușkela des Hundes isolirte Glykogen. Denn alle auf die specifische Drehung und die Beziehung des Glykogenes zu dem resultirenden Zucker bei der Inversion bezüglichen Werthe stimmen genau überein. Es muss hinzugefügt werden, dass nach den Untersuchungen von J. Nerking ${ }^{1}$ ) und von Mme Z. GatinGružewska ${ }^{2}$ ) dasselbe für das Glykogen des Pferdes und Hundes festgestellt worden ist. Man darf wohl annehmen, dass das Glykogen aller Thiere und aller Organe dieselbe Substanz ist. Die Arteigenschaft fehlt also dem Glykogen. Da nach Allem, was wir wissen, auch der Traubenzucker in den Säften der Thiere überall derselbe Stoff ist, dürfen wir weiter folgern, dass die thierischen Kohlehydrate die Eigenschaft der Arteigenthümlichkeit nicht besitzen. - - Es liegen aber ferner keine Thatsachen vor, dass die Fettarten, welche in den Organen verschiedener. Thiere vorkommen, sich durch Arteigenthümlichkeiten auszeichnen. - Nur für die Eiweissstoffe gilt die merkwürdige Thatsache, dass jeder Thierart be: sondere Eiweissarten eigenthümlich sind.

Weil die Eiweissmoleküle verschiedener Thiere eine verschiedene chemische Constitution haben, ist es selbstverständlich, dass sie auch wieder verschiedene Spaltungsproducte liefern werden. Also nicht das Eiweiss an sich, sondern auch die Derivate desselben müssen Arteigenthümlichkeit aufweisen. Ich würde es deshalb in hohem Grade wahrscheinlich finden, dass in den Gallensäuren, die ja Arteigenthümlichkeit zeigen, nicht bloss die stickstoff- und schwefel-

1) J. Ne rking, Ueber die elementare Zusammensetzung und das Invertierungsvermögen des Glykogenes. Dieses Archiv Bd. 85 S. 329.1901.

2) Mme Z. Gatin-Gružewska, Das reine Glykogen. Dieses Archiv Bd. 102 S. 579, 580.1904. 
378 Eduard Pfiügẹr: Meine Methode der quant. Analyse des Glykogenes etc.

haltige Componente, d. h. das Glykokoll und das Taurin, sondern auch die stickstoff- und schwefelfreie - nämlich die Cholalsäure aus dem Gefüge des Eiweissmoleküles stammen, weil diese Säure Arteig̣enthümlichkeiten besitzt. In der Erkenntniss der Ausnahmestellung der Eiweissstoffe, die sie mit Rücksicht auf die Arteigenthümlichkeit zeigen, wird uns ein höchst werthvolles Mittel an die Hand gegeben, um den Beziehungen der verschiedenen Stoffe des Thierkörpers näher zu treten. -

Selbstverständlich bin ich der Ansicht, dass hier zwei ganz verschiedene Kreise von Arteigenthümlichleiten in Betracht kommen.

Der eine Kreis umfasst diejenigen Thatsachen, welche sich auf die verschiedenen im Thierreich auftretenden Arten der Eiweissstoffe und ihrer Derivate beziehen - im Gegensatz zu den Fetten, Koblehydraten und Mineralstoffen, deren Molekülen die Arteigenthümlichkeit fehlt. -

Der zweite Kreis ist dadurch gekennzeichnet, dass unabhängig von der chemischen Qualität der Moleküle das Mischungsverhältniss derselben bei verschiedenen Thierarten ein sehr versehiedenes sein kann.

Obwohl diese Thatsachen einfach genug erscheinen, komme ich hier nochmals darauf zurück, weil Niemand vor mir die grosse Bedeutung dieser Gebiete erkannt hat. Denn in den neuesten Lehrbüchern der physiologischen Chemie, wie bei Thierfelder, Hammarsten, ja sogar in den "Ausblicken" von Abderhalden finde ich nirgends eine Andeutung. 